\title{
Pacific
}

Journal of

Mathematics

\section{A FUNCTIONAL CALCULUS FOR UNBOUNDED GENERALIZED SCALAR OPERATORS ON BANACH SPACES}

DRAGOLJUb KeČKIĆ AND ĐORĐE KRTINIĆ 


\title{
A FUNCTIONAL CALCULUS FOR UNBOUNDED GENERALIZED SCALAR OPERATORS ON BANACH SPACES
}

\author{
DragoluUb KEČKIĆ AND ĐORĐE KRTINIĆ
}

We define weak and ultraweak functional calculus and construct using the Fourier transform technique an ultraweak functional calculus for an unbounded $k$-tuple of commuting generalized scalar operators $T_{j}$ acting on a Banach space $X$. This functional calculus comprises the functions from the subset

$$
F_{\alpha}=\left\{f: \mathbb{R}^{k} \rightarrow \mathbb{C} \mid \hat{f} \text { is a measure such that } \int_{\mathbb{R}^{k}}\left(1+|t|^{\alpha}\right) d|\hat{f}|<\infty\right\},
$$

of Sobolev space $W_{\infty}^{\alpha}(\mathbb{R})$, where $\alpha>0$. It also contains some unbounded functions. We also give examples and related results.

\section{Introduction}

A functional calculus for an operator $A$ is an (in some sense) continuous homomorphism $\Phi: \mathscr{A} \rightarrow \mathscr{B}$, where $\mathscr{A}$ is a topological algebra of functions and $\mathscr{B}$ is a topological algebra of operators, such that $\Phi(1)=e$ is the unit of the algebra $\mathscr{B}$ and $\Phi(x)=A$, where $x$ denotes the identity function. Also, it is expected that the algebra $\mathscr{A}$ is generated by 1 and $x$, that is, that polynomials are dense in $\mathscr{A}$ (in the topology of $\mathscr{A})$.

A familiar example of a functional calculus is the analytic functional calculus on Banach algebras. It is the homomorphism $\Phi: \mathscr{H} \rightarrow \mathscr{C}$, where $\mathscr{C}$ is a Banach algebra, $A \in \mathscr{C}$ and $\mathscr{H}$ is an algebra of functions holomorphic in some neighborhood of $\sigma(A)$ (in the topology generated by uniform convergence on compact sets), defined by

$$
\Phi(f)=\frac{1}{2 \pi i} \int_{\Gamma} f(\lambda)(\lambda e-A)^{-1} d \lambda=f(A),
$$

where $\Gamma \subset \operatorname{Dom}(f)$ and $\sigma(A) \Subset$ int $\Gamma$.

This mapping is continuous in the natural way.

MSC2000: primary 46H25, 46H30, 47A60, 47L60; secondary 47L10, 47D06.

Keywords: functional calculus, Fourier transform, unbounded operators.

Supported by MNZZS grant number 174034, Serbia. 
Another example of a functional calculus is described by the spectral theorem. Let $\mathscr{H}$ be a Hilbert space and $A \in B(\mathscr{H})$ be a normal operator. Then

$$
L^{\infty}(\sigma(A)) \ni f \rightarrow \int_{\sigma(A)} f(\lambda) d E_{\lambda}=f(A)
$$

( $E_{\lambda}$ is the spectral measure) defines a homomorphism $\Phi: L^{\infty} \rightarrow B(\mathscr{H})$ that is continuous in the norm, because $\|f\|_{\infty}=\|f(A)\|$ and because the pointwise convergence of $f_{n}$ to $f$ implies $f_{n}(A) \rightarrow f(A)$, strongly ("continuity").

From these examples, we can see that if a functional calculus is defined for a wide class of operators, then the algebra of functions is very small. Analogously, if the algebra of functions is wide, then we must have stronger conditions on the operators.

In this note we will use the Fourier transform technique, which is not new and has been applied in many papers. McIntosh and Pryde [1987] developed the functional calculus for commuting $n$-tuples of bounded generalized scalar operators and functions that have Fourier transform in $L_{s}^{1}=\left\{f\left|\int\right| f(t) \mid\left(1+|t|^{s}\right) d t<+\infty\right\}$. A more general result was obtained by Andersson and Berndtsson [2002]. The case of a single generator of a bounded strongly continuous group was treated in [Balabane et al. 1993]. For more details and references, see [deLaubenfels 1994].

A similar idea of using Laplace transform for generators of bounded or polynomially bounded semigroups was used in [deLaubenfels and Jazar 1999] and [deLaubenfels 1995].

Another direction of development of functional calculi uses the Cauchy-Green integral formula applied to the almost holomorphic extension of a test function. The initial work of Dynkin [1972] was followed by the articles [Helffer and Sjöstrand 1989; Andersson 2003; Andersson and Sjöstrand 2004; Andersson et al. 2006]. The results obtained by this approach are mostly consequences of our results. In particular, Andersson [2003] showed that the functional calculus can be extended via the Cauchy-Green formula to the algebra of $C^{\infty}$ functions with an additional condition at infinity if and only if the corresponding group is polynomially bounded, although a wider class of operators admits functional calculus for test functions. Andersson, Samuelsson and Sandberg [2006] observed that for any proper $f \in C^{\infty}\left(\mathbb{R}^{m}, \mathbb{R}^{k}\right)$, the set $\left\{g \circ f \mid g \in \mathscr{D}\left(\mathbb{R}^{k}\right)\right\}$ is contained in $D\left(\mathbb{R}^{m}\right)$; they then used this fact to construct the functional calculus (with test functions) for $f(A)$, where $f$ is a proper $C^{\infty}$ function and $A$ is a Helffer-Sjöstrand operator, that is, an operator with real spectrum and rationally bounded resolvent on compacts.

After basic definitions in Section 2, including those of weak and ultraweak functional calculus, we introduce in Section 3 the algebra $F_{\alpha}$ and in Section 4 the algebra $F_{\alpha, \text { loc }}$. In Section 4 , we construct the functional calculus for the $n$ tuple of commuting generalized scalar operators and functions from $F_{\alpha, \text { loc. In }}$ 
Section 5, we prove that the support of such functional calculus coincides with the spectrum of some operator closely related to the initial $n$-tuple and acting on the Clifford algebra derived from the initial Banach space. In Section 6, we compare the (ultra) weak functional calculus with other axiomatic approaches and find it to be more comprehensive. In other words, for very wide algebra of functions we obtain a more comprehensive theory. As shown in [Andersson 2003], this class of operators is exactly the one obtained by using the Cauchy-Green integral formula. In Section 7, we discuss relationship between some distinct definitions of commutativity of unbounded operators. In Section 8, we give some examples and comments.

\section{Basic definitions}

In this paper $\mathbb{R}^{k} \ni t=\left(t_{1}, \ldots, t_{k}\right)$ and $T=\left(T_{1}, \ldots, T_{k}\right)$ are $k$-tuples of numbers and operators, respectively. We define $t \cdot T:=t_{1} T_{1}+\cdots+t_{k} T_{k}$, the "inner product", and $|t|:=\sqrt{t_{1}^{2}+\cdots+t_{k}^{2}}$, the Euclidean norm.

The next definition is a modification of definition given in [Vasilescu 1982].

Definition 2.1. Let $T=\left(T_{1}, \ldots, T_{k}\right)$ be a $k$-tuple of closed densely defined operators on a Banach space $X$. We call the ordered couple $(\mathscr{A}, \Phi)$ a weak functional calculus based on $\mathbb{R}^{k}$ if

(1) $\mathscr{A}$ is a normed algebra of functions defined on $\mathbb{R}^{k}$ with a topology $\tau$, such that

(a) $\tau$ is weaker than the norm topology;

(b) the addition is continuous with respect to $\tau$;

the multiplication is separately continuous with respect to $\tau$;

(c) the test functions $\mathscr{D}$ are included and dense in $\mathscr{A}$;

(d) embedding $\mathscr{D} \hookrightarrow \mathscr{A}$ is continuous;

(2) $\Phi: \mathscr{A} \rightarrow B(X)$ is homomorphism, which is norm continuous and continuous with respect to $\tau$ and the weak topology on $B(X)$;

(3) for every polynomial $p\left(t_{1}, \ldots, t_{k}\right)$ of degree $m$ and for every sequence of the test functions $\vartheta_{n}$ tending to 1 in the natural way (increasingly and uniformly on compacts) we have $\Lambda\left(\Phi\left(\vartheta_{n} p\right) x\right) \rightarrow \Lambda\left(p\left(T_{1}, \ldots, T_{k}\right) x\right)$ for all $x \in \bigcap_{\sum m_{j}=m} \mathscr{D}\left(T_{1}^{m_{1}} \cdots T_{k}^{m_{k}}\right)$ and all $\Lambda \in X^{*}$.

Remark 2.2. In the condition (1), we expect continuity of multiplication only by coordinates. It would be to much to expect full continuity. Indeed, in that case from condition (2) it would follow that if $A_{n} \rightarrow A$ and $B_{n} \rightarrow B$ weakly, then $A_{n} B_{n} \rightarrow A B$ weakly, which is not true.

To extend the functional calculus to a wide set of unbounded functions, we need another condition, included in the following definition. 
Definition 2.3. Let $T=\left(T_{1}, \ldots, T_{k}\right)$ be a $k$-tuple of closed densely defined operators on a Banach space $X$. We call the ordered couple $(\mathscr{A}, \Phi)$ an ultraweak functional calculus based on $\mathbb{R}^{k}$ if it is a weak functional calculus and if the algebra $A$ satisfies the following condition:

For an arbitrary test function $0 \leqslant \varphi \leqslant 1$ equalling 1 in some neighborhood of zero, and for any $f \in \mathscr{A}$, there holds $\varphi(x / n) f(x) \rightarrow f(x)$ as $n \rightarrow \infty$, in the topology of $\mathscr{A}$.

We will prove the existence of an ultraweak functional calculus when $\mathscr{A}$ is the subspace

$$
F_{\alpha}=\left\{f: \mathbb{R}^{k} \rightarrow \mathbb{C} \mid \hat{f} \text { is a measure such that } \int_{\mathbb{R}}\left(1+|t|^{\alpha}\right) d|\hat{f}|<\infty\right\}
$$

of the Sobolev space $W_{\infty}^{\alpha}\left(\mathbb{R}^{k}\right)$, where $\alpha=\sum_{j=1}^{k} \alpha_{j}$ is a positive real number. We assume that all operators $T_{j}$ are closed and densely defined, $\sigma\left(T_{j}\right) \subseteq \mathbb{R}$ and

$$
\left\|e^{i t T_{j}}\right\|=O\left(|t|^{\alpha_{j}}\right) \quad \text { as } t \rightarrow \infty .
$$

Also, we assume that $T_{j}$ are mutually commuting, which means that the corresponding groups $e^{i t T_{j}}$ and $e^{i s T_{k}}$ mutually commute for any $s, t \in \mathbb{R}$ and any $j, k$.

Remark 2.4. We should specify what is meant by $e^{i t T_{j}}$, because the set

$$
\left\{x \in X \mid x \in \bigcap_{n \geqslant 1} \mathscr{D}\left(T^{n}\right) \wedge \sum_{n=1}^{\infty} \frac{\left\|T^{n} x\right\|}{n !}<\infty\right\}
$$

(the so-called analytical vectors) need not be dense in $X$, according to Nelson's theorem.

In this paper, $e^{i t T}$ is a one-parameter group $C(t)$, the solution of the abstract Cauchy problem

$$
\frac{d}{d t} C(t)=i T C(t), \quad C(0)=I .
$$

One can prove the existence of solution of Equation (2-2) under different kinds of conditions (for example, the Hille-Yoshida conditions). Also, it is possible to give an estimate of asymptotic behavior of that solution; see [deLaubenfels 1991; 1993b; 1993a].

Remark 2.5. The condition $\sigma\left(T_{j}\right) \subseteq \mathbb{R}$ is actually superflous. Namely, Andersson and Berndtsson [2002, Lemma 1.2] showed that the condition (2-1) implies that the joint spectrum of the $n$-tuple of commuting bounded operators is a subset of $\mathbb{R}^{n}$. The proof presented in their Remark 1 is still valid if we omit their boundedness. However, many conditions that ensure the existence of the solution of the abstract Cauchy problem include the reality of spectrum. 
Remark 2.6. One can define commutativity of unbounded operators in various ways. For instance, one can assume there is a dense subspace on which all products $T_{i} T_{j}$ are well-defined and $T_{i} T_{j}=T_{j} T_{i}$. In the literature, one also finds that the commutativity of the pair of unbounded operators is defined as the commutativity of their resolvents. In Section 7, we compare different commutativity conditions.

Remark 2.7. From the Hille-Yoshida theorem (see [Dunford and Schwartz 1958]) we can conclude that for a closed, densely defined operator $A$, with real spectrum such that $\left\|(A-\lambda I)^{-1}\right\| \leqslant 1 /|\lambda|^{m}$ for nonreal $\lambda$, Equation (2-2) has a solution satisfying

$$
\|C(t)\|=o\left(e^{\varepsilon t}\right) \text { for all } \varepsilon>0 .
$$

A natural question arises: Can we derive a polynomial estimate of $C(t)$ from (2-3)? The following example shows that this is not possible.

Example 2.8. There is an operator for which (2-1) does not hold, and its asymptotic behavior is weaker than exponential (that is, $e^{i t A}=o\left(e^{\varepsilon|t|}\right)$ as $|t| \rightarrow \infty$ for every $\varepsilon>0$ ).

Let $E$ be the vector space of entire functions such that there exists $C \in \mathbb{R}$ such that $|f(z)| \leqslant C e^{\sqrt{|z|}}$. One can easily see that $E$ is the Banach space if the norm $\|f\|$ of a function $f$ is defined as the smallest constant $C$, for which the inequality above holds (that is, $\|f\|=\sup _{z \in \mathbb{C}}|f(z)| / e^{\sqrt{|z|}}$ ). Since

$$
f^{\prime}(z)=\frac{1}{2 \pi i} \int_{|\xi|=1} \frac{f(\xi+z)}{\xi^{2}} d \xi,
$$

it follows that operator $A=-i d / d z$ is a bounded linear operator on $E$. Hence

$$
\left(e^{i t A} f\right)(z)=\left(e^{t d / d z} f\right)(z)=f(z+t) .
$$

Since

$$
\frac{\left|\left(e^{i t A} f\right)(z)\right|}{e^{\sqrt{z}}}=\frac{|f(z+t)|}{e^{\sqrt{|z+t|}}} \cdot \frac{e^{\sqrt{|z+t|}}}{e^{\sqrt{|z|}}} \leqslant\|f\| \cdot e^{\sqrt{|z|+|t|}-\sqrt{|z|}} \quad \text { for every } f \in E,
$$

it follows that $\left\|e^{i t A}\right\|=O\left(e^{\sqrt{|t|}}\right)$ as $|t| \rightarrow \infty$. Let $f_{0}(z)=2 \sum_{n=0}^{\infty} z^{n} /(2 n)$ !. From the above it follows that $\left\|e^{i t A} f_{0}\right\| /\left\|f_{0}\right\| \leqslant e^{\sqrt{|t|}}$. Since for $x, t \geqslant 0$, we have

$$
\begin{aligned}
\frac{\left(e^{i t A} f_{0}\right)(x)}{e^{\sqrt{x}}} & =\frac{2 \sum_{n=0}^{\infty}(x+t)^{n} /(2 n) !}{e^{\sqrt{x}}}=\frac{2 \operatorname{ch}(\sqrt{x+t})}{e^{\sqrt{x}}} \\
& =e^{\sqrt{x+t}-\sqrt{x}}+e^{-\sqrt{x+t}-\sqrt{x}},
\end{aligned}
$$

it follows that $\left\|e^{i t A} f_{0}\right\| \sim e^{\sqrt{|t|}}$ as $t \rightarrow \infty$; therefore $\left\|e^{i t A} f_{0}\right\| \sim e^{\sqrt{|t|}}$ as $t \rightarrow \infty$. 


\section{The algebra $F_{\alpha}$}

Definition 3.1. Let $\alpha$ be a positive real number, and let $\mathcal{M}_{\alpha}\left(\mathbb{R}^{k}\right)$ be the space of all Borel measures on $\mathbb{R}^{k}$ such that $\int_{\mathbb{R}^{k}}\left(1+|t|^{\alpha}\right) d|\mu|<\infty$ with the norm $\|\mu\|_{\alpha}=$ $\int_{\mathbb{R}^{k}}\left(1+|t|^{\alpha}\right) d|\mu|$. Let $F_{\alpha}=\left\{f \in L^{\infty}\left(\mathbb{R}^{k}\right) \mid \hat{f} \in \mathcal{M}_{\alpha}\right\}$ be the normed space with the norm $\|f\|_{F_{\alpha}}=\|\hat{f}\|_{M_{\alpha}}$.

Since $F_{\alpha}=\check{M}_{\alpha}$, it follows that $F_{\alpha}$ is the Banach space with the norm $\|\cdot\|_{F_{\alpha}}$; that is

$$
|\check{\mu}(x)|=\frac{1}{(2 \pi)^{k / 2}} \cdot\left|\int_{\mathbb{R}^{k}} e^{i x \cdot t} d \mu(t)\right| \leqslant \frac{1}{(2 \pi)^{k / 2}} \cdot\|\mu\|_{\mathcal{M}_{\alpha}}<\infty .
$$

Let $\tau$ be the topology on $F_{\alpha}$ generated by the weak* topology on $\mathcal{M}_{\alpha}\left(\mathbb{R}^{k}\right) \subseteq$ $\left(C_{b, \alpha}\left(\mathbb{R}^{k}\right)\right)^{*}$, where $C_{b, \alpha}\left(\mathbb{R}^{k}\right)$ is the space of continuous functions such that $f(t)$ is $O\left(|t|^{\alpha}\right)$ as $|t| \rightarrow \infty$, with the norm $\|f\|_{C_{b, \alpha}}=\inf \left\{M|| f(t) \mid \leqslant M \cdot\left(1+|t|^{\alpha}\right)\right\}$. It means that the subbase of the neighborhoods of zero are

$$
\mathscr{B}(g, \varepsilon)=\left\{f \in F_{\alpha}|| \int_{\mathbb{R}^{k}} g d \hat{f} \mid<\varepsilon\right\} \quad \text { for } g \in C_{b, \alpha}\left(\mathbb{R}^{k}\right) \text { and } \varepsilon>0 ;
$$

see [Rudin 1973, Chapter 1].

Remark 3.2. Andersson and Berndtsson [2002] studied more general algebras $\mathscr{A}_{h}$, where $h: \mathbb{R}^{k} \rightarrow \mathbb{R}$ is a positive, continuous, subadditive function that is increasing on the rays from origin, $h(0)=0$, and $h(t)=o(|t|)$ as $t \rightarrow \infty$. The algebra $F_{\alpha}$ is a special case of $\mathscr{A}_{h}$, where $h(x)=\log \left(1+|x|^{\alpha}\right)$. The last function is not subadditive, but satisfies a somewhat weaker condition $h(x+y) \leq \log 2^{\alpha}+h(x)+h(y)$.

Theorem 3.3. The space $F_{\alpha}$ is an algebra.

Proof. $F_{\alpha}$ is a linear space, obviously. Let us prove that $f, g \in F_{\alpha}$ implies $f \cdot g \in F_{\alpha}$. The inequality

$$
1+|s+t|^{\alpha} \leqslant 2^{\alpha}\left(1+|s|^{\alpha}\right)\left(1+|t|^{\alpha}\right)
$$

holds. Indeed, for $|s| \leqslant|t|$ we have $1+|s+t|^{\alpha} \leqslant 1+(2|t|)^{\alpha} \leqslant 2^{\alpha}\left(1+|t|^{\alpha}\right) \leqslant$ $2^{\alpha}\left(1+|t|^{\alpha}\right)\left(1+|s|^{\alpha}\right)$ and similarly for $|s| \geqslant|t|$. Therefore, if $f, g \in F_{\alpha}$, then $\hat{f}$ and $\hat{g}$ are finite measures such that $\int_{\mathbb{R}^{k}}\left(1+|t|^{\alpha}\right) d|\hat{f}|<+\infty$ and $\int_{\mathbb{R}^{k}}\left(1+|t|^{\alpha}\right) d|\hat{g}|<+\infty$. Also $\widehat{f \cdot g}=\hat{f} * \hat{g}$ is well-defined and finite by the finiteness of $\hat{f}$ and $\hat{g}$, and we have

$$
\begin{aligned}
\int_{\mathbb{R}^{k}}\left(1+|t|^{\alpha}\right) d|\hat{f} * \hat{g}| & \leqslant \int_{\mathbb{R}^{k}} \int_{\mathbb{R}^{k}}\left(1+|s+t|^{\alpha}\right) d|\hat{f}|(s) d|\hat{g}|(t) \\
& \leqslant 2^{\alpha} \int_{\mathbb{R}^{k}}\left(1+|s|^{\alpha}\right) d|\hat{f}|(s) \int_{\mathbb{R}^{k}}\left(1+|t|^{\alpha}\right) d|\hat{g}|(t) \\
& =2^{\alpha}\|f\|_{F_{\alpha}}\|g\|_{F_{\alpha}} .
\end{aligned}
$$


Remark 3.4. Although such a space arises rarely in the literature, one can define the Sobolev space $W_{\infty}^{\alpha}$ as the space of all functions $f: \mathbb{R}^{k} \rightarrow \mathbb{R}$ such that its fractional partial derivative $(-\Delta)^{\alpha / 2} f$ belongs to $L^{\infty}$. Here fractional derivative $(-\Delta)^{\alpha / 2}$ is defined via Fourier transform. However $W_{\infty}^{\alpha} \supset F_{\alpha}$ (that is, this inclusion is proper) since Fourier transforms of functions from $F_{\alpha}$ have always continuous fractional partial derivative.

Remark 3.5. One can, also, define the space $F_{\left(\alpha_{1}, \ldots, \alpha_{n}\right)}$ consisting of all functions $f$ whose Fourier transform $\hat{f}$ is a measure such that $\int_{\mathbb{R}^{k}}\left(1+\left|t_{1}\right|^{\alpha_{1}} \cdots \cdot\left|t_{n}\right|^{\alpha_{n}}\right) d|\hat{f}|$ is finite. A simple application of the mean inequality shows that $F_{\alpha} \subset F_{\left(\alpha_{1}, \ldots, \alpha_{n}\right)}$. From the following example we can see that this inclusion is strict.

Example 3.6. Let $n=2$ and $\left(\alpha_{1}, \alpha_{2}\right)=(1,1)$. Then we have

$$
F_{(1,1)}=\left\{f\left|\int_{\mathbb{R}^{2}}(1+|x y|) d\right| \hat{f} \mid<+\infty\right\} \quad \text { and } \quad F_{2}=\left\{f \mid \int_{\mathbb{R}^{2}}\left(1+x^{2}+y^{2}\right) d \hat{f}<+\infty\right\} .
$$

Consider the function $\psi$ defined as the inverse Fourier transform of the function

$$
(x, y) \mapsto \frac{1}{1+|x y|} \cdot \frac{1}{1+x^{2}+y^{2}} \cdot \frac{1}{\log ^{2}\left(1+\left(x^{2}+y^{2}\right) / 2\right)} .
$$

We claim that $\psi \in F_{(1,1)} \backslash F_{2}$. Indeed

$$
\int_{\mathbb{R}^{2}}(1+|x y|) d \hat{\psi}=\int_{\mathbb{R}^{2}} \frac{1}{1+x^{2}+y^{2}} \cdot \frac{1}{\log ^{2}\left(1+\left(x^{2}+y^{2}\right) / 2\right)} d x d y<+\infty,
$$

whereas

$$
\begin{aligned}
\int_{\mathbb{R}^{2}}\left(1+x^{2}+y^{2}\right) d \hat{\psi} & =\int_{\mathbb{R}^{2}} \frac{1}{1+|x y|} \cdot \frac{1}{\log ^{2}\left(1+\left(x^{2}+y^{2}\right) / 2\right)} d x d y \\
& \geqslant \int_{|x| \geqslant|y|} \frac{1}{1+|x y|} \cdot \frac{1}{\log ^{2}\left(1+x^{2}\right)} d x d y \\
& =2 \int_{-\infty}^{+\infty} \frac{1}{\log ^{2}\left(1+x^{2}\right)} \cdot \int_{0}^{|x|} \frac{d|y|}{1+|x y|} d x \\
& =2 \int_{-\infty}^{+\infty} \frac{d x}{|x| \log \left(1+x^{2}\right)}=+\infty
\end{aligned}
$$

However, space $F_{\left(\alpha_{1}, \ldots, \alpha_{n}\right)}$, although wider, need not be an algebra.

Example 3.7. Let $n=2$ and $\left(\alpha_{1}, \alpha_{2}\right)=(1,1)$. The functions $\varphi(x, y)=\sin x^{2}$ and $\psi(x, y)=\sin y^{2}$ are in $F_{(1,1)}$ since they are bounded and have bounded second order mixed derivatives $\partial^{2} \varphi / \partial x \partial y$ and $\partial^{2} \psi / \partial x \partial y$. However, their product has no bounded second order mixed derivatives. Hence $F_{(1,1)}$ is not an algebra.

Theorem 3.8. The set $\mathscr{D}$ is dense in the space $F_{\alpha}$ (with the topology $\tau$ ) and the embedding $\mathscr{D} \hookrightarrow F_{\alpha}$ is continuous. 
Proof. The sequence $\left([-n, n]^{k}\right)_{n \geqslant 1}$ is an increasing sequence of sets and we have $\bigcup_{n \in \mathbb{N}}[-n, n]^{k}=\mathbb{R}^{k}$. Therefore $|\mu|\left([-n, n]^{k}\right) \rightarrow|\mu|\left(\mathbb{R}^{k}\right)$ as $n \rightarrow \infty$ for $\mu \in M_{\alpha}$. If $g \in C_{b, \alpha}\left(\mathbb{R}^{k}\right)$ and $M=\sup _{x \in \mathbb{R}^{k}}|g(x)|$, it follows that there is $n \in \mathbb{N}$ such that $|\mu|\left(\mathbb{R}^{k} \backslash[-n, n]^{k}\right)<\varepsilon / M$. Thus $\check{\mu}_{n} \in \check{\mu}+\mathscr{B}(g, \varepsilon)$, where $\mu_{n}(E)=\mu\left(E \cap[-n, n]^{k}\right)$ for every measurable set $E$. It follows that it is enough to prove the first part of our statement for inverse images $f \in F_{\alpha}$ of functions with compact support.

Let $\mu$ be a measure with compact support. Let $h_{j}(x)=j^{k} h(j x)$ be an approximative unit on $\mathbb{R}^{k}$ (that is, a sequence $\left(h_{j}\right)_{j \in \mathbb{N}}$ where $h \in \mathscr{D}\left(\mathbb{R}^{k}\right), h \geqslant 0$ and $\int_{\mathbb{R}^{k}} h(x) d x=1$; see [Rudin 1973, 6.31]). Then we have $h_{j} * \mu \in \mathscr{S}$ (because $D^{\alpha}\left(h_{j} * \mu\right)=\left(D^{\alpha} h_{j}\right) * \mu$, and since $h_{j}$ and $\mu$ have compact support, so does $h_{j} * \mu$; here $\mathscr{Y}$ is the Schwartz class). If $g_{1}(t)=g(-t)$, it follows that

$$
\begin{aligned}
\left(h_{j} * \mu\right)(g) & =\left(\left(h_{j} * \mu\right) * g_{1}\right)(0) \\
& =\left(h_{j} *\left(\mu * g_{1}\right)\right)(0) \rightarrow \delta *\left(\mu * g_{1}\right)(0)=\mu * g_{1}(0)=\mu(g)
\end{aligned}
$$

by [Rudin 1973, 6.30]. Therefore $h_{j} * \mu \rightarrow \mu$ as $j \rightarrow \infty$ in the weak* topology on $M_{\alpha}$. Hence $\mathscr{Y}$ is dense in the weak* topology on $M_{\alpha}$, so it is also dense in the space $F_{\alpha}$ with the topology $\tau$, because $\hat{S}=S$.

Therefore, it is enough to prove that $\mathscr{D}$ is dense in $\mathscr{S}$.

Since $\mathscr{D}$ is dense in $\mathscr{S}$ in the topology of the space $\mathscr{T},{ }^{1}$ for $\varphi \in \mathscr{Y}$ there exists $\left(\varphi_{n}\right)_{n \geqslant 1}$ such that $\varphi_{n} \rightarrow \varphi$ in $\mathscr{Y}$ as $n \rightarrow \infty$.

Let $g \in C_{b, \alpha}\left(\mathbb{R}^{k}\right)$. Then $\hat{g} \in \mathscr{S}^{\prime}$ (see [Rudin 1973, 7.14(d)]) and therefore $\int_{\mathbb{R}^{k}} g\left(\hat{\varphi}_{n}-\hat{\varphi}\right) d x=\int_{\mathbb{R}^{k}}\left(\varphi_{n}-\varphi\right) d \hat{g} \rightarrow 0$. That means that $\varphi_{n} \rightarrow \varphi$ as $n \rightarrow \infty$ in the topology $\tau$.

The last part of the statement is trivial for test functions with support in some fixed compact set. Therefore by [Rudin 1973, Theorem 6.6], it is true for all $\mathscr{D}$.

Lemma 3.9. Pointwise addition and multiplication are continuous in $\left(F_{\alpha}, \tau\right)$.

Proof. Let $\lim _{\Gamma} f_{\gamma}=f$ and $\lim _{\Gamma} g_{\gamma}=g$ in the topology of $F_{\alpha}$, where $\Gamma$ is a net. Then $\int_{\mathbb{R}^{k}} h d \hat{f}_{\gamma} \rightarrow \int_{\mathbb{R}^{k}} h d \hat{f}$ and $\int_{\mathbb{R}^{k}} g d \hat{g}_{\gamma} \rightarrow \int_{\mathbb{R}^{k}} h d \hat{g}$ for arbitrary $h \in C_{b, \alpha}\left(\mathbb{R}^{k}\right)$. Then we have

$$
\int_{\mathbb{R}^{k}} h d\left(\widehat{f_{\gamma}+g_{\gamma}}\right)=\int_{\mathbb{R}^{k}} h d \hat{f}_{\gamma}+\int_{\mathbb{R}^{k}} h d \hat{g}_{\gamma} \rightarrow \int_{\mathbb{R}^{k}} h d \hat{f}+\int_{\mathbb{R}^{k}} h d \hat{g}=\int_{\mathbb{R}^{k}} h d \widehat{(\hat{f+g})}
$$

for $\gamma \in \Gamma$.

To prove the continuity of multiplication, it is enough to consider $f_{\gamma} \rightarrow 0$, and fixed $g$. Let $\mathscr{B}_{h_{1}, \ldots, h_{n}, \varepsilon_{1}, \ldots, \varepsilon_{n}}=\left\{\varphi \in F_{\alpha}|| \int_{\mathbb{R}^{k}} h_{j} d \hat{\varphi} \mid<\varepsilon_{j}, j=1, \ldots, n\right\}$ be an

${ }^{1}$ Analogously to the procedure from the beginning of this proof, let $\left(h_{j}\right)_{j \in \mathbb{N}}$ be an approximative unit, such that $h(x) \leqslant 1$ and $h \equiv 1$ in some neighborhood of 0 ; then $\mathscr{D} \ni h_{j} *\left(\chi_{[-n, n]^{k}} \cdot \varphi\right) \rightarrow \varphi$ in the topology of $\mathscr{Y}$ 
arbitrary basic neighborhood of zero generated by $h_{j} \in C_{b, \alpha}$. Using inequality (3-1), it is easy to check that the functions

$$
\psi_{j}(x)=\int_{\mathbb{R}^{k}} h_{j}(x+y) d \hat{g}(y)
$$

also belong to $C_{b, \alpha}$. If $f_{\gamma} \in \mathscr{B}_{\psi_{1}, \ldots, \psi_{n}, \varepsilon_{1}, \ldots, \varepsilon_{n}}$, then $f_{\gamma} \cdot g \in \mathscr{B}_{h_{1}, \ldots, h_{n}, \varepsilon_{1}, \ldots, \varepsilon_{n}}$. Indeed,

$$
\left|\int_{\mathbb{R}^{k}} h_{j} d \hat{f}_{\gamma} * \hat{g}\right|=\left|\iint_{\mathbb{R}^{k} \times \mathbb{R}^{k}} h_{j}(x+y) d \hat{g}(y) d \hat{f}_{\gamma}(x)\right|=\left|\int_{\mathbb{R}^{k}} \psi_{j} d \hat{f}_{\gamma}\right|<\varepsilon_{j} .
$$

In the general case, we have $f_{\gamma} \cdot g-f g=\left(f_{\gamma}-f\right) \cdot g$, and we can apply the special case.

From Theorems 3.3 and 3.8 and Lemma 3.9 it follows that the space $\left(F_{\alpha}, \tau\right)$ (from Definition 3.1) satisfies condition (1) of Definition 2.1.

Proposition 3.10. Let $\varphi$ be a test function such that $\varphi \equiv 1$ on some neighborhood of zero, and such that $0 \leqslant \varphi \leqslant 1$. Let $\chi_{n}(x)=\varphi(x / n)$. Then $f \chi_{n} \rightarrow f$ in the topology of the space $F_{\alpha}($ as $n \rightarrow \infty)$.

Proof. We need to prove $\int_{\mathbb{R}^{k}} g d \widehat{f \chi_{n}} \rightarrow \int_{\mathbb{R}^{k}} g d \hat{f}$ as $n \rightarrow \infty$ for any $g \in C_{b, \alpha}\left(\mathbb{R}^{k}\right)$.

Let $F_{n}(t, s)=\int_{\mathbb{R}^{k}} g(t+s) d \hat{\chi}_{n}(s)$. Since $\hat{\chi}_{n}(s) \rightarrow \delta$ (the Dirac distribution) in the topology of $F_{\alpha}$ as $n \rightarrow \infty,{ }^{2}$ we have $\lim _{n \rightarrow \infty} F_{n}(t, s)=g(t)$.

Since

$\int_{\mathbb{R}^{k}} g d \widehat{f \chi_{n}}=\int_{\mathbb{R}^{k}} g d\left(\hat{f} * \hat{\chi}_{n}\right)=\int_{\mathbb{R}^{k}} \int_{\mathbb{R}^{k}} g(s+t) d \hat{f}(t) d \hat{\chi}_{n}(s)=\int_{\mathbb{R}^{k}} F_{n}(t, s) d \hat{f}(t)$, and $\hat{\chi}_{n}(s)=n^{k} \hat{\phi}(n s)$ (see [Rudin 1973, Theorem 7.4]), our result follows from the dominated convergence theorem, because

$$
\begin{aligned}
\left|F_{n}(t, s)\right| & \leqslant \int_{\mathbb{R}^{k}}|g(t+s)| d|\hat{\chi}(s)| \\
& \leqslant \int_{\mathbb{R}^{k}} n^{k}\left(1+|s+t|^{\alpha}\right) d \hat{\phi}(n s) \\
& \leqslant 2^{\alpha}\left(1+|t|^{\alpha}\right) \int_{\mathbb{R}^{k}} n^{k}\left(1+|s|^{\alpha}\right)(1+|n s|)^{-\alpha-n-1} d s \\
& =2^{\alpha}\left(1+|t|^{\alpha}\right) \int_{\mathbb{R}^{k}} \frac{\left(1+|u / n|^{\alpha}\right)}{(1+|u|)^{\alpha+n+1}} d u \\
& \leqslant 2^{\alpha}\left(1+|t|^{\alpha}\right) \int_{\mathbb{R}^{k}} \frac{\left(1+|u|^{\alpha}\right)}{(1+|u|)^{\alpha+n+1}} d u \\
& \leqslant \text { const } \cdot\left(1+|t|^{\alpha}\right) .
\end{aligned}
$$

${ }^{2} \hat{\chi}_{n}(s)$ belongs to the Schwartz class $\mathscr{S}$ and tends to $\delta$ in the topology of $\mathscr{G}$; see Theorem 3.8. 
We used that $g \in C_{b, \alpha}\left(\mathbb{R}^{k}\right), \hat{\phi} \in \mathscr{S}\left(\right.$ so $\left|\hat{\phi}^{\prime}(s)\right| \leqslant$ const $\cdot(1+|s|)^{-m}$ for any natural $\left.m\right)$, $|u / n| \leqslant|u|$ for positive integer $n$, and inequality (3-1).

The preceding lemma asserts that the algebra $F_{\alpha}$ satisfies the condition from the definition of the ultraweak functional calculus.

\section{The construction of the functional calculus}

Following the technique from [McIntosh and Pryde 1987] we define the functional calculus using the Fourier transform, that is, by formula

$$
W_{\infty}^{\alpha} \supseteq F_{\alpha} \ni f \mapsto \Phi(f)=f(T)=\frac{1}{(2 \pi)^{k / 2}} \cdot \int_{\mathbb{R}^{k}} e^{-i t \cdot T} d \mu_{\hat{f}},
$$

where the measure $\mu_{\hat{f}}$ is the Fourier transformation of the function $f$ (namely, $\hat{f}(t)=(2 \pi)^{-k / 2} \cdot \int_{\mathbb{R}^{k}} e^{-i t s} f(s) d s$ can be divergent for some $t$, but this formula defines a complex Borel measure $\mu_{\hat{f}}$ and we have $\left.\int_{-\infty}^{\infty}\left(1+|t|^{\alpha}\right) d\left|\mu_{\hat{f}}\right|<\infty\right)$.

From now on, we shall abbreviate $\mu_{\hat{f}}$ to $\hat{f}$.

Theorem 4.1. Suppose $T=\left(T_{1}, \ldots, T_{k}\right)$ is a $k$-tuple of closed, densely defined operators such that all $e^{i t T_{j}}$ exist as solutions of the corresponding abstract Cauchy problem (2-2), such that

$$
\left\|e^{i t T_{j}}\right\|=O\left(|t|^{\alpha_{j}}\right) \quad \text { as } t \rightarrow \infty .
$$

Also, we assume that $T_{j}$ are mutually commuting, which means that the corresponding groups $e^{i t T_{j}}$ and $e^{i s T_{k}}$ mutually commute for any $s, t \in \mathbb{R}$ and any $j, k$.

Let $X$ be a reflexive Banach space and let $\alpha=\sum_{j} \alpha_{j} \geqslant 0$. A homomorphism $\Phi: F_{\alpha} \rightarrow B(X)$ is defined by formula (4-1). The integral in (4-1) exists as a weak integral and $\Phi$ is $(\tau, \omega)$ continuous, where $\tau$ is the topology on $F_{\alpha}$ as the subspace of the dual space of $C_{b, \alpha}$ and $\omega$ is the weak topology on $B(X)$.

Proof. Let $x \in X$ and $\Lambda \in X^{*}$. Function $t \mapsto \Lambda\left(e^{i t \cdot T} x\right)$ is weakly continuous. Also, we have

$$
\left|\int_{\mathbb{R}^{k}} \Lambda\left(e^{i t \cdot T} x\right) d \mu_{\hat{f}}\right| \leqslant \int_{\mathbb{R}^{k}}\|\Lambda\|\left(1+|t|^{\alpha}\right)\|x\| d\left|\mu_{\hat{f}}\right| \leqslant\|\Lambda\| \cdot\|x\| \cdot\|f\|_{\alpha} ;
$$

hence (4-1) exists as a weak integral.

The mapping $\Phi$ is obviously linear; also, we have

$$
\begin{aligned}
\Phi(f) \cdot \Phi(g) & =\frac{1}{(2 \pi)^{k}} \cdot \int_{\mathbb{R}^{k}} \int_{\mathbb{R}^{k}} e^{i(t+s) \cdot T} d \mu_{\hat{f}}(s) d \mu_{\hat{g}}(t) \\
& =\frac{1}{(2 \pi)^{k / 2}} \cdot \int_{\mathbb{R}^{k}} e^{i r \cdot T} d \mu_{\hat{f} * \hat{g}}(r)=\frac{1}{(2 \pi)^{k / 2}} \cdot \int_{\mathbb{R}^{k}} e^{i r \cdot T} d \mu_{\widehat{f g}}(r)=\Phi(f g),
\end{aligned}
$$

where $r=s+t$. 
Finally, we need to prove the continuity.

Let $S_{\Lambda, x, \varepsilon}=\{A|| \Lambda(A x) \mid<\varepsilon\}$ be a subbase set in $B(X)$, let $f_{0} \in F_{\alpha}$, and let $A_{f}=(2 \pi)^{-k / 2} \cdot \int_{\mathbb{R}^{k}} e^{i t \cdot T} d \mu_{\hat{f}}(t) \in B(X)$ for $f \in F_{\alpha}$.

Let

$$
V=\left\{f\left|\frac{1}{(2 \pi)^{k / 2}} \cdot\right| \int_{\mathbb{R}^{k}} \Lambda\left(e^{i t \cdot T} x\right) d \mu \widehat{f-f_{0}} \mid<\varepsilon\right\} .
$$

Since $t \mapsto \Lambda\left(e^{i t \cdot T} x\right)$ is continuous, $V$ is a neighborhood in $F_{\alpha}$. Since

$$
\Lambda\left[\left(A_{f}-A_{f_{0}}\right) x\right]=\frac{1}{(2 \pi)^{k / 2}} \cdot \int_{\mathbb{R}^{k}} \Lambda\left(e^{i t \cdot T} x\right) d \mu_{\widehat{f-f_{0}}}(t)<\varepsilon,
$$

it follows that $\Phi\left(f_{0}+V\right) \subseteq A_{f_{0}}+S_{\Lambda, x, \varepsilon}$.

Theorem 4.2. Let $p\left(t_{1}, t_{2}, \ldots, t_{k}\right)$ be a polynomial of degree $m$ and let $\vartheta_{n}$ be a sequence of test functions tending to 1 increasingly and uniformly on compacts. Then for all $\phi \in \bigcap_{\sum m_{j}=m} \operatorname{Dom} \prod_{j} T_{j}^{m_{j}}$ we have $\Phi\left(\vartheta_{n} p\right) \phi \stackrel{w}{\rightarrow} p\left(T_{1}, T_{2}, \ldots, T_{k}\right) \phi$ as $n \rightarrow \infty$.

Proof. Function $t \mapsto e^{i t \cdot T}$ is strongly and weakly differentiable and satisfies (2-2). Let $D_{j}=-i \partial / \partial t_{j}$ and let $\delta$ be the Dirac distribution. Since

$$
D_{j}^{m}(\hat{1})=D_{j}^{m}\left((2 \pi)^{k / 2} \delta\right)=(-1)^{m} \widehat{t}_{j}^{m}
$$

(for all $m \in \mathbb{N}_{0}$ ) and $\vartheta_{n}$ has compact support, from the definition of the distribution and [Rudin 1973, 6.37], we have

$$
\begin{aligned}
& T_{1}^{m_{1}} \cdots T_{k}^{m_{k}} \phi=\frac{1}{(2 \pi)^{k / 2}} \cdot \int_{\mathbb{R}^{k}} T_{1}^{m_{1}} \cdots \cdots T_{k}^{m_{k}} e^{i t \cdot T} \phi d\left((2 \pi)^{k / 2} \cdot \delta\right) \\
& =\lim _{n \rightarrow \infty} \frac{1}{(2 \pi)^{k / 2}} \cdot \int_{\mathbb{R}^{k}} D_{1}^{m_{1}} \cdots D_{k}^{m_{k}}\left(e^{i t \cdot T}\right) \phi d \mu_{\left(\hat{1} * \widehat{\vartheta_{n}}\right)} \\
& =(-1)^{m_{1}+\cdots+m_{k}} \lim _{n \rightarrow \infty} \frac{1}{(2 \pi)^{k / 2}} \cdot \int_{\mathbb{R}^{k}} e^{i t \cdot T} \phi d \mu_{\left(D_{1}^{m_{1}} \ldots \ldots D_{k}^{m_{k}}(\hat{1}) * \widehat{\vartheta_{n}}\right)} \\
& =(-1)^{\sum_{j} m_{j}} \lim _{n \rightarrow \infty} \frac{1}{(2 \pi)^{k / 2}} \cdot \int_{\mathbb{R}^{k}} e^{i t \cdot T} \phi d \mu_{\left(\left((-1)^{\sum_{j} m_{j}} t_{1}^{m} \widehat{m_{1} \ldots t_{k}^{m}}\right) * \widehat{\vartheta_{n}}\right)} \\
& =\lim _{n \rightarrow \infty} \frac{1}{(2 \pi)^{k / 2}} \cdot \int_{\mathbb{R}^{k}} e^{i t \cdot T} \phi d \mu_{\left(t_{1}^{m_{1}}, \ldots \cdot t_{k}^{m_{k}} \cdot \vartheta_{n}\right)},
\end{aligned}
$$

which proves our statement in the case where $p\left(t_{1}, \ldots, t_{k}\right)=t_{1}^{m_{1}} \cdots t_{k}^{m_{k}}$ is a monomial. In general case, the result easily follows by linearity of $\Phi$.

The previous results can be summarized:

Formula (4-1) defines an ultraweak functional calculus.

Next, we shall see that the possibility of constructing the operator $f(T)$ does not depend on the behavior of the function $f$ at infinity. In fact, it depends on the local properties of the function $f$. 
Throughout the rest of this section, $\varphi_{n}$ (and also $\psi_{n}$ and $\chi_{n}$ ) will always denote an increasing sequence of test functions with range contained in $[0,1]$, and such that $\left\{t \in \mathbb{R}^{k} \mid \varphi_{n}(t)=1\right\}$ form an increasing sequence of sets whose union covers all $\mathbb{R}^{k}$, unless $\varphi_{n}$ is specified otherwise. Such sequences we shall call exhausting.

Proposition 4.3. Let $X_{n}$ denote the linear space $\operatorname{Im}\left(\chi_{n}(T)\right)$. Let $E_{0}=\bigcup_{n=1}^{+\infty} X_{n}$. Then $X_{n}$ is invariant for all operators $f(T)$ and $E=\mathscr{L}\left(E_{0}\right)$ is dense in $X$.

Proof. Let $x \in X_{n}$, that is, $x=\chi_{n}(T) y$. Then

$$
f(T) x=f(T) \chi_{n}(T) y=\chi_{n}(T) f(T) y \in X_{n} .
$$

Since $\chi_{n} \rightarrow 1$ in the topology of $F_{\alpha}$, we have $x=w-\lim x_{n}=w-\lim \chi_{n}(A) x$ for any $x \in X$. Therefore the set $E_{0}$ is weakly dense, and its linear span $E$ is also weakly dense. For linear spaces weak and strong closures coincide.

Definition 4.4. We say that $f \in F_{\alpha, \text { loc }}$ if $f \varphi \in F_{\alpha}$ for any $\varphi \in \mathscr{D}$.

Proposition 4.5. $F_{\alpha} \subseteq F_{\alpha, \text { loc }}$ and $F_{\alpha, \text { loc }}$ is an algebra.

Proof. The first assertion follows from $\mathscr{D} \cdot F_{\alpha} \subseteq F_{\alpha} \cdot F_{\alpha} \subseteq F_{\alpha}$. Let $f, g \in F_{\alpha \text {, loc }}$ and let $\varphi \in \mathscr{D}$ be arbitrary. Choose another $\psi \in \mathscr{D}$ such that $\varphi \psi=\varphi$, that is, a function equals 1 in the neighborhood of the support of $\varphi$. Then $f \varphi, g \psi \in F_{\alpha}$ and $f g \varphi=f \varphi \cdot g \psi \in F_{\alpha}$.

Proposition 4.6. Let $f \in F_{\alpha, l o c}$, let $\chi_{n}$ be some exhausting sequence and let $x \in E$. The sequence $\left(f \cdot \chi_{n}\right)(T) x$ converges strongly and its limit does not depend on the choice of an exhausting sequence.

Proof. Let $x \in X_{k}$. Then $x=\psi_{k}(A) y$ for some $y \in X$. Pick a positive integer $n_{0}$ such that $\chi_{n_{0}}=1$ on supp $\psi_{k}$. For $n \geqslant n_{0}$ we have $\left(f \cdot \chi_{n}\right)(T) x-\left(f \cdot \chi_{n_{0}}\right)(T) x=$ $\left(\left(f \cdot\left(\chi_{n}-\chi_{n_{0}}\right) \cdot \psi_{k}(T)\right) y=0\right.$, implying that $\left(f \cdot \chi_{n}\right)(T) x$ is constant for $n \geqslant n_{0}$. Therefore, this sequence is constant also for $x \in E$.

To prove the independence, choose another exhausting $\psi_{n}$, and set

$$
y=\lim _{n \rightarrow+\infty}\left(f \chi_{n}\right)(T) \text { and } z=\lim _{n \rightarrow+\infty}\left(f \psi_{n}\right)(T) .
$$

For an arbitrary $\theta \in \mathscr{D}$, we have

$$
\theta(T) y=\lim _{n \rightarrow+\infty} \theta(T)\left(f \chi_{n}\right)(T) x=(\theta f)(T) x,
$$

and analogously $\theta(T) z=(\theta f)(T) x$. Thus for any $\theta \in \mathscr{D}$, we have $\theta(T)(y-z)=0$. If $\theta$ runs through $\theta_{n}(x)=\varphi(x / n)$, then $\theta_{n}(T) \rightarrow I$ weakly, implying $y-z=0$.

Definition 4.7. Let $f \in F_{\alpha, \text { loc }}$ and let $x \in E$. We define $f(T) x:=\lim \left(f \cdot \chi_{n}\right)(T) x$. Thus, we defined the map $f \mapsto f(T)$

Proposition 4.8. The map defined in Definition 4.7 is linear and multiplicative. 
Proof. The linearity is obvious. Further, let us note that the sequence $\left(f \chi_{n}\right)(T) x$ is not only convergent but constant for $n \geqslant n_{0}$. Let $\chi_{n}$ be an arbitrary exhausting sequence and let $x=\psi_{k}(T) y \in X_{k}$. Choose another exhausting sequence $\varphi_{n}$ such that $\chi_{n} \varphi_{n}=\chi_{n}$. Then we have

$$
(f g)(T) x=\lim _{n \rightarrow+\infty}\left(f \chi_{n}\right)(T)\left(g \varphi_{n}\right)(T) \psi_{k}(T) y .
$$

Since all the operators $\left(f \chi_{n}\right)(T),\left(g \varphi_{n}\right)(T)$ and $\psi_{k}(T)$ commute, we see that both $\left(g \varphi_{n}\right)(T) y$ and $\left(f \chi_{n}\right)(T) y$ belong to $X_{k}$. Therefore $\left(g \varphi_{n}\right)(T) y$ and $g(T) y$ (as its limit) are in the domain of $f(T)$. In other words, the operator $f(T) g(T)$ is welldefined on $X_{n}$. By changing roles of $f$ and $g$, we conclude that $g(T) f(T)$ is also well-defined on $X_{n}$. From (4-2), we get $(f g)(T)=f(T) g(T)=g(T) f(T)$.

Proposition 4.9. For any $f \in F_{\alpha, \text { loc }}$, the operator $f(T)$ is closable.

Proof. Let $x_{n} \rightarrow 0$, and let $f(T) x_{n} \rightarrow y$. We have

$$
y=w-\lim _{n \rightarrow+\infty} \chi_{n}(T)=w-\lim _{n \rightarrow+\infty} \lim _{m \rightarrow+\infty} \chi_{n}(T) f(T) x_{m}=0
$$

since both $\chi_{n}(T)$ and $\left(\chi_{n} f\right)(T)$ are bounded.

\section{The support of the functional calculus}

Definition 5.1. The support of the functional calculus is the smallest closed set $\operatorname{supp} \Phi \subseteq \mathbb{R}^{k}$ such that if $f \equiv g$ on $\operatorname{supp} \Phi$, then $\Phi(f)=\Phi(g)$.

First we need some definitions and facts concerning Clifford algebras.

Definition 5.2. Suppose $F$ is an $m$-dimensional real vector space $F$ with a basis $\left\{e_{j} \mid 1 \leqslant j \leqslant m\right\}$. We define the Clifford algebra $\mathscr{C l}(F)$ as a vector space with basis $\left\{e_{S} \mid S \subseteq\{1,2, \ldots, m\}\right\}$, with multiplication arising from identifications $e_{\varnothing}=1$, $e_{\{j\}}=e_{j}$ and rules

$$
e_{j}^{2}=-1 \quad \text { and } \quad e_{j} e_{k}=-e_{k} e_{j} \text { if } k \neq j
$$

Remark 5.3. The rules (5-1) can be easily extended to the basis of $\mathscr{C l}(F)$ if we write $e_{j_{1}} e_{j_{2}} \cdots e_{j_{s}}$ for $e_{S}=e_{\left\{j_{1}, j_{2}, \ldots, j_{s}\right\}}$, and further by linearity to whole $\mathscr{C l}(F)$.

Remark 5.4. For an $m$-dimensional real vector space $F$, we will write $\mathscr{C l}(\mathrm{m})$ for $\mathscr{C l}(F)$. It is well known that $\mathscr{C l}(1)$ is isomorphic to the field of complex numbers, and that $\mathscr{C l}(2)$ to the quaternions. For further details on Clifford algebras, see [Brackx et al. 1982; Ryan 2003]. 
Definition 5.5. Let $X$ be a Banach space. The corresponding Banach module over $\mathscr{C l}(m)$ is simply $X_{(m)}=X \otimes_{\mathbb{R}} \mathscr{C} l(m)=\left\{\sum_{S \subseteq\{1,2, \ldots, m\}} u_{S} e_{S} \mid u_{S} \in X\right\}$, with the norm

$$
\left\|\sum_{S \subseteq\{1,2, \ldots, m\}} u_{S} e_{S}\right\|=\left(\sum_{S \subseteq\{1,2, \ldots, m\}}\left\|u_{S}\right\|^{2}\right)^{1 / 2} .
$$

Definition 5.6. Let $T_{0}, T_{1}, \ldots, T_{m}$ be an $(m+1)$-tuple of operators acting on a Banach space $X$ (bounded or unbounded). The operator $T_{\mathrm{cl}}=\sum_{j=0}^{m} T_{j} e_{j}$ is defined by $T_{\mathrm{cl}}\left(\sum_{S} u_{S} e_{S}\right)=\sum_{j=0}^{m} \sum_{S} T_{j} u_{S} e_{j} e_{S}$.

The norm of $T_{\mathrm{cl}}$ is $\left\|T_{\mathrm{cl}}\right\|=\sup _{\|u\| \leqslant 1}\left\|T_{\mathrm{cl}} u\right\|$.

Also we can define the resolvent set and the spectrum of $T_{\mathrm{cl}}$ by $\rho\left(T_{\mathrm{cl}}\right)=\left\{\lambda \in \mathbb{R}^{m+1} \mid \lambda I-T_{\mathrm{cl}}\right.$ is invertible $\} \quad$ and $\quad \sigma\left(T_{\mathrm{cl}}\right)=\mathbb{R}^{m+1} \backslash \rho\left(T_{\mathrm{cl}}\right)$.

Proposition 5.7. (a) We have $\|u \lambda\| \leqslant 2^{m / 2}\|u\|\|\lambda\|$ for all $u \in X_{m}$ and $\lambda \in \mathscr{C l}(m)$. (b) $\sigma\left(T_{\mathrm{cl}}\right) \subseteq\left\{\lambda \in \mathbb{R}^{m+1}|| \lambda \mid \leqslant \sqrt{m+1} \cdot\left\|T_{\mathrm{cl}}\right\|\right\}$.

Proof. Direct calculation.

Proposition 5.8. Let $T_{0}, T_{1}, \ldots, T_{m}$ be an $(m+1)$-tuple of commuting unbounded operators. By commutativity we mean that there is a dense set $D \subseteq X$ such that $T_{j} T_{k} x=T_{k} T_{j} x$ for all $x \in D$ and $j, k$. Also, let $T_{\mathrm{cl}}=\sum_{j=0}^{m} T_{j} e_{j}$.

(a) The following conditions are mutually equivalent:

(i) $T_{\mathrm{cl}}$ is invertible.

(ii) $\left(\sum_{j} T_{j}^{2}\right) e_{0}$ is invertible.

(iii) $\sum_{j} T_{j}^{2}$ is invertible in $B(X)$.

(b) $\sigma\left(T_{\mathrm{cl}}\right)=\left\{\lambda \in \mathbb{R}^{m} \mid \sum_{j}\left(T_{j}-\lambda_{j}\right)^{2}\right.$ is not invertible $\}$.

Proof. If $\sum_{j} T_{j}^{2}$ is invertible in $B(X)$, then $T_{\mathrm{cl}}^{-1}=\left(\sum_{j} T_{j}^{2}\right)^{-1} \cdot\left(T_{0}-\sum_{j>0} T_{j} e_{j}\right)$, so (iii) implies (i).

Since $\left(T_{0}-\sum_{j>0} T_{j} e_{j}\right) U=U T_{\mathrm{cl}}$, where $U\left(\sum_{j} u_{S} e_{S}\right)=\sum_{j}(-1)^{|S|} u_{S} e_{S}$, it follows that $\left(T_{0}-\sum_{j>0} T_{j} e_{j}\right)$ is invertible; hence $\left(\sum_{j} T_{j}^{2}\right) e_{0}=T_{\mathrm{cl}}\left(T_{0}-\sum_{j>0} T_{j} e_{j}\right)$ is invertible, that is, (i) implies (ii).

That (ii) is equivalent to (iii) is obvious. Part (b) follows from the equivalence of (i) and (iii).

Theorem 5.9. The support of the weak functional calculus defined in Definition 2.1 is equal to $\sigma\left(\sum_{i=1}^{m} T_{i} e_{i}\right)$.

Proof. Let $n$ be an odd integer, let $n \geqslant \max \{m, 2\}$, and let $T=\sum_{i=1}^{m} T_{i} e_{i}$. By Proposition 5.8, and since supp $\Phi \subseteq \mathbb{R}^{m}$, it is enough to prove that $\sigma(T)=\operatorname{supp} \Phi$. If $f=\sum_{S} f_{S} e_{S}$, with $\Phi_{(n)}(f)=\sum_{S} \Phi\left(f_{S}\right) e_{S}$, we can extend $\Phi$ to a homomorphism $\Phi_{(n)}: \mathscr{A} \otimes \mathbb{R} \mathbb{R}_{(n)} \rightarrow B(X)_{(n)}$, which is continuous with respect to $\tau$ and the weak topology on $B(X)_{(n)}$ and supp $\Phi_{(n)}=\operatorname{supp} \Phi$. 
Let $\lambda \notin \operatorname{supp} \Phi$ and let $\vartheta_{i} \in \mathscr{D}$ such that $\vartheta_{i}(\lambda)=0$ and $\operatorname{supp} \vartheta_{i} \rightarrow \operatorname{supp} \Phi$ (increasingly and uniformly on compacts). Then the function $|x-\lambda|^{-n-1} \overline{(x-\lambda)}$ is well-defined (with value 0 for $x=\lambda$ ) and the function $(x-\lambda)|x-\lambda|^{n-1}$ is polynomial (since $n$ is odd). Therefore we have

$$
\begin{aligned}
& (T-\lambda I)|T-\lambda I|^{n-1} \cdot \lim _{i \rightarrow \infty} \Phi_{(n)}\left(\vartheta_{i} \cdot|x-\lambda|^{-n-1} \overline{(x-\lambda)}\right) \\
& \quad=\lim _{i \rightarrow \infty} \Phi_{(n)}\left(\vartheta_{i} \cdot(x-\lambda)|x-\lambda|^{n-1}\right) \Phi_{(n)}\left(\vartheta_{i} \cdot|x-\lambda|^{-n-1} \overline{(x-\lambda)}\right) \\
& \quad=\lim _{i \rightarrow \infty} \Phi_{(n)}\left(\vartheta_{i}^{2}\right)=I .
\end{aligned}
$$

Analogously, $\lim _{i \rightarrow \infty} \Phi_{(n)}\left(\vartheta_{i} \cdot|x-\lambda|^{-n-1} \overline{(x-\lambda)}\right) \cdot(T-\lambda I)|T-\lambda I|^{n-1}=I$, implying that $T-\lambda I$ is invertible, that is, $\lambda \notin \sigma(T)$.

For the opposite direction, suppose $f$ is in $\mathscr{D}$ and satisfies supp $f \cap \sigma(T)=\varnothing$. Let

$$
F(x)= \begin{cases}(T-x I)^{-1}|T-x I|^{-n+1} \Phi_{(n)}(f) & \text { for } x \notin \sigma(T) \\ \Phi_{(n)}\left(f(t) \cdot|t-x|^{-n-1} \overline{(t-x)}\right) & \text { for } x \notin \operatorname{supp} f .\end{cases}
$$

Since supp $f \cap \sigma(T)=\varnothing$, it follows that the function $F$ is defined for all $x$. If $x \notin \sigma(T), x \notin \operatorname{supp} f$, and $\vartheta_{i}$ is an element of $\mathscr{D}$ such that $\vartheta_{i} \equiv 1$ on supp $f$ and $\vartheta_{i}$ tends to 1 increasingly and uniformly on compacts, then

$$
\begin{aligned}
& (T-x I)|T-x I|^{n-1} \cdot \Phi_{(n)}\left(f(t) \cdot|t-x|^{-n-1} \overline{(t-x)}\right) \\
& \quad=\lim _{i \rightarrow \infty} \Phi_{(n)}\left(\vartheta_{i} \cdot(t-x)|t-x|^{n-1}\right) \cdot \Phi_{(n)}\left(f(t) \cdot|t-x|^{-n-1} \overline{(t-x)}\right) \\
& \quad=\lim _{i \rightarrow \infty} \Phi_{(n)}\left(\vartheta_{i} f\right)=\Phi_{(n)}(f),
\end{aligned}
$$

implying $F$ is well-defined.

Taking into account [McIntosh and Pryde 1987, Examples 5.3 and 5.4], we see the function $F$ is an entire monogenic function. Since $f(t) \cdot|t-x|^{-n-1} \overline{(t-x)} \rightrightarrows 0$ as $|x| \rightarrow \infty$, it follows that

$$
\Phi_{(n)}\left(f(t) \cdot|t-x|^{-n-1} \overline{(t-x)}\right) \stackrel{w}{\rightarrow} 0 \quad \text { in } B(X)_{(n)},
$$

so the range of $\Phi_{(n)}$ is weakly bounded. But in locally convex space every weakly bounded set is originally bounded (see [Rudin 1973, Theorem 3.18] - actually, this theorem is consequence of the Banach-Alaoglu theorem, the Hahn-Banach theorem and the Banach-Steinhaus theorem). So the $\Phi_{(n)}\left(f(t) \cdot|t-x|^{-n-1} \overline{(t-x)}\right)$ are norm bounded. By Liouville's theorem for monogenic functions (see [McIntosh and Pryde 1987, Theorem 5.1]), $F$ is a constant function. However, a constant sequence of operators tending weakly to 0 must be identically equal to zero, that is, $F \equiv 0$. Hence $\Phi_{(n)}(f)=0$, that is, $\operatorname{supp} \Phi_{(n)} \subseteq \sigma(T)$.

Remark 5.10. McIntosh, Pryde and Ricker [McIntosh et al. 1988] proved that for the $k$-tuple of commuting bounded generalized scalar operators, the spectrum 
$\sigma(T)$ coincides with many known joint spectra of the $\left(T_{1}, \ldots, T_{k}\right)$, for instance Harte and Taylor spectrum. An interesting question for further research is to find whether this statement holds for a $k$-tuple of unbounded operators.

\section{Comparison of different definitions of functional calculi}

We defined the notion of weak and ultraweak functional calculus. There are at least two other definitions of functional calculi.

Definition 6.1 [deLaubenfels 1995]. Let $T$ be an unbounded operator on a Banach space $X$ and let $\mathscr{F}$ be an algebra of functions. Also, let $f_{1}(x)=x$ be the identical function, let $f_{0}(x) \equiv 1$, and let $g_{\lambda}(z)$ denote the mapping $z \mapsto(z-\lambda)^{-1}$. The mapping $\mathscr{F} \ni f \mapsto f(T) \in B(X)$ is a $\mathscr{F}$-functional calculus if it is a homomorphism and if the following conditions are satisfied:

(i) $f(T) T \subseteq T f(T)=\left(f \cdot f_{1}\right)(T)$ whenever $f$ and $f \cdot f_{1}$ belong to $\mathscr{F}$.

(ii) $f_{0}(T)=I_{X}$ whenever $f_{0} \in \mathscr{F}$.

(iii) $T-\lambda I$ is injective and $\left(g_{\lambda}^{k}\right)(T)=(T-\lambda I)^{-k}$ whenever $g_{\lambda}^{k} \in \mathscr{F}$.

Definition 6.2 [Andersson et al. 2006]. We call a continuous multiplicative mapping $A: \mathscr{D}\left(\mathbb{R}^{n}\right) \rightarrow B(X)$ is a hyperoperator on $\mathbb{R}^{n}$, writing $A \in H_{\mathscr{D}\left(\mathbb{R}^{n}\right)}(X)$, if

(i) $D_{A}=\bigcup \operatorname{Im} A(\phi)$ is dense in $X$, and

(ii) $N=\bigcap \operatorname{Ker} A(\phi)=\{0\}$.

Definition 6.3 [Andersson et al. 2006]. Let $c=(c, D)$ be a linear operator mapping the dense subspace $D$ of $X$ into itself. Moreover, assume that $c$ is closable and that there is a linear and multiplicative mapping $\mathscr{E}\left(\mathbb{R}^{n}\right) \rightarrow \mathrm{七}(D)$ that extends the trivial one on polynomials, and such that $h_{k}(c) x \rightarrow h(c) x$ for $x \in D$ if $h_{k} \rightarrow h$ in $\mathscr{E}\left(\mathbb{R}^{n}\right)$. Then we say that $c$, or rather $(c, D)$, is a weak hyperoperator.

In the preceding definition, $\mathscr{E}\left(\mathbb{R}^{n}\right)$ is the algebra of all differentiable functions on $\mathbb{R}^{n}$, which possess an asymptotic expansion $f(x) \sim \sum_{k=0}^{\infty} a_{k} x^{-k}($ when $x \rightarrow \infty$ ), with $a_{k} \in \mathbb{C}$, in the sense that for every $N \in \mathbb{N}$

$$
f(x)=\sum_{k=0}^{N} a_{k} x^{-k}+x^{-N-1} r_{N+1}(x) \text { for }|x|>1,
$$

where $r_{N+1}(x)$ is bounded with all its derivatives (for more details see [Andersson and Sjöstrand 2004]) and $€(D)$ denotes the set (not the space!) of all closable operators on $D$.

Proposition 6.4. Any ultraweak functional calculus is an A-functional calculus in the sense of Definition 6.1. 
Proof. (i) Let $x \in \mathscr{D}(T)$ and let $\chi_{n}=\varphi(x / n)$, where $\phi$ is a test function equal to 1 in some neighborhood of zero and satisfies $0 \leqslant \varphi \leqslant 1$. By the definition of the ultraweak functional calculus, we have $\mathscr{D} \ni f f_{1} \chi_{n} \rightarrow f f_{1}$ in the topology of $\mathscr{A}$. Then $\left(f f_{1} \chi_{n}\right)(T) \rightarrow f f_{1}(T)$ weakly. So, for $x$ in the domain of $T$ we have

$$
f(T) T x=w-\lim f(T)\left(f_{1} \chi_{n}\right)(T) x=w-\lim \left(f f_{1} \chi_{n}\right)(T) x=\left(f f_{1}\right)(T) x,
$$

implying $f(T) T \subseteq\left(f f_{1}\right)(T)$. On the other hand, for any $x$ such that $f(T) x$ belongs to the domain of $T$, we have

$$
\left(f f_{1}\right)(T) x=w-\lim \left(f_{1} \chi_{n}\right)(T) f(T) x=T f(T) x .
$$

The set of such vectors $x$ contains the set $E$ from Proposition 4.3, and therefore it is dense in $X$. By continuity, we get $T f(T)=\left(f f_{1}\right)(T)$.

(ii) Let $f_{0} \in \mathscr{A}$. Choose a sequence $\chi_{n} \in \mathscr{D}$ tending to $f_{0}$ in the topology of $\mathscr{A}$. (Note that the definition of the weak functional calculus contains the condition that $\mathscr{D}$ is dense in $\mathscr{A}$ in the topology of $\mathscr{A}$.) Then $f_{0}(T) x=w-\lim \chi_{n}(T) x=x$ by the definition of the ultraweak functional calculus.

(iii) Let $g_{\lambda}^{k} \in \mathscr{A}$. We have $(z-\lambda)^{k} g_{\lambda}(z)=1$. Let $\chi_{n} \in \mathscr{D}$ be an exhausting sequence. By the definition of the ultraweak functional calculus and by the condition (3) of Definition 2.1 (applied to the polynomial $t \mapsto t$ ), we have

$$
x=w-\lim \left(\chi_{n}\left(f_{1}-\lambda\right)^{k} g_{\lambda}^{k}\right)(T) x=(T-\lambda I)^{k} g_{\lambda}(T)^{k} x=g_{\lambda}^{k}(T)(T-\lambda I)^{k} x .
$$

Proposition 6.5. The restriction of any weak functional calculus to $\mathscr{D}$ is a hyperoperator.

Proof. We only have to prove the conditions (i) and (ii) from Definition 6.2. But this easily follows from condition (3) of Definition 2.1 applied to the polynomial $f_{0}(x) \equiv 1$.

Remark 6.6. We think that an interesting question for further investigation is to find the relationship between an ultraweak functional calculus and the notion of weak hyperoperator.

\section{On commutativity conditions}

For an $n$-tuple of unbounded operators, it is difficult to find a commutativity condition that ensures a reasonable theory. Any of the following four properties can be used to define of commutativity of two unbounded operators on a Banach space:

(P1) There exists a dense space $\mathscr{D} \subseteq X$ such that $T S x=S T x$ for any $x \in \mathscr{D}$.

(P2) Suppose that there exists a $\mathscr{D}(\mathbb{R})$ functional calculus for both $T$ and $S$. For any $f, g \in \mathscr{D}(\mathbb{R})$, we have $f(T) g(S)=g(S) f(T)$. 
(P3) Suppose that $T$ and $S$ are generators of strongly continuous groups $e^{i t T}$ and $e^{i t S}$, respectively. For any $t, s \in \mathbb{R}$, we have $e^{i t T} e^{i s S}=e^{i s S} e^{i t T}$.

(P4) For any $\lambda \in \rho(T)$ and $\mu \in \rho(S)$, we have

$$
(T-\lambda I)^{-1}(S-\mu I)^{-1}=(S-\mu I)^{-1}(T-\lambda I)^{-1} .
$$

In the general case all of these properties except (P1) might be meaningless, since an unbounded operator need not have a $\mathscr{D}(\mathbb{R})$ functional calculus, a solution of the corresponding abstract Cauchy problem, or a nonempty resolvent set.

However, if $S$ and $T$ are generalized scalar operators, (P1)-(P4) make sense and are mutually equivalent.

Proposition 7.1. (P1) implies (P4).

Proof. Obvious.

Proposition 7.2. (P3) implies (P1) if $S$ and $T$ are generalized scalar operators.

Proof. For such $S$ and $T$, we constructed the ultraweak functional calculus and extended it to $F_{\alpha, \text { loc }}$. The function $f(x, y)=x y$ belongs to $F_{\alpha, l o c}$, so the operator $f(T, S)$ is defined on the linear span of $E$ (defined in Proposition 4.3). Examining the proof of Theorem 4.2, we see that on the set $E$ both products $T S$ and $S T$ are equal to $f(T, S)$.

Proposition 7.3. (P3) implies (P2) if $S$ and $T$ are generalized scalar operators.

Proof. For the pair $(S, T)$, we constructed a functional calculus for a wider algebra than $\mathscr{D}\left(\mathbb{R}^{2}\right)$, and choosing a function of the form $f(x) g(y)$, we obtain the result.

Proposition 7.4. (P4) implies (P3) if $S$ and $T$ are generalized scalar operators.

Proof. $S$ and $T$ are generalized scalar operators that are generators of strongly continuous groups, so this statement can be derived following the standard proof of the Hille-Yoshida theorem; see [Dunford and Schwartz 1958, Theorem VIII.1.13]. The spectrum of $i T$ is on the imaginary line, so $\lambda \in \rho(i T)$ for all $\lambda>0$. It follows that $B_{\lambda}=-i \lambda\left[I-\lambda(\lambda I-i T)^{-1}\right]$ (for $\lambda>0$ ) are bounded. Also, $i T x=\lim _{\lambda \rightarrow \infty} B_{\lambda} x$ for all $x \in \mathscr{D}(i T)=\mathscr{D}(T)$ and $\lim _{\lambda \rightarrow \infty} e^{t B_{\lambda}} x$ exists for all $x \in \mathscr{D}(i T)=\mathscr{D}(T)$ (of course, the value of this limit is $\left.e^{i t T} x\right)$. Analogously, if $C_{\lambda}=-i \lambda\left[I-\lambda(\lambda I-i C)^{-1}\right]$ (for $\lambda>0$ ), we have $i S x=\lim _{\lambda \rightarrow \infty} C_{\lambda} x$ for all $x \in \mathscr{D}(i S)=\mathscr{D}(S)$ and $\lim _{\lambda \rightarrow \infty} e^{s C_{\lambda}} x$ exists for all $x \in \mathscr{D}(i S)=\mathscr{D}(S)$.

Finally, from (P4) (the commutativity of resolvents) we have the commutativity of $B_{\lambda}$ and $C_{\lambda}$ (for all $\lambda>0$ ), and, passing to $\lambda \rightarrow \infty$, we get (P3).

Proposition 7.5. (P2) implies (P4) if $S$ and $T$ are generalized scalar operators.

Proof. The function $g_{\lambda}(x)=1 /(x-\lambda)$ belongs to $F_{\alpha}$ for all $\alpha$ and for $\operatorname{Im} \lambda \neq 0$. Indeed, it is easy to check that $g_{\lambda}$ is the inverse Fourier transform of the function 
$F(x)=i \sqrt{2 \pi} e^{-i \lambda x} H(-x)$ for $\operatorname{Im} \lambda>0$. Similarly, it is the inverse Fourier transform of the function $F(x)=-i \sqrt{2 \pi} e^{-i \lambda x} H(x)$ for $\operatorname{Im} \lambda<0$. Here $H(x)$ is the Heaviside function, equal to zero if $x<0$ and to 1 otherwise. Hence $g_{\lambda} \in F_{\alpha}$, and $\left\|g_{\lambda}\right\|_{F_{\alpha}}=\sqrt{2 \pi}\left(1 /|\operatorname{Im} \lambda|+\Gamma(\alpha+1) /|\operatorname{Im} \lambda|^{\alpha}\right)$.

If $\varphi_{n}(x)=\varphi(x / n)$, where $\varphi$ is a test function valued in $[0,1]$ and equal to 1 in a neighborhood of zero, then the sequence of test functions $\psi_{n, \lambda}=\varphi_{n} \cdot g_{\lambda} \rightarrow g_{\lambda}$ as $n \rightarrow+\infty$ in the topology $\tau$ defined in Definition 3.1, and therefore $\psi_{n, \lambda}(S) \rightarrow$ $g_{\lambda}(S)=(S-\lambda I)^{-1}$ weakly, by Proposition 6.4. Similarly $\psi_{m, \mu}(T) \rightarrow(T-\mu I)^{-1}$ weakly. From property (P2) we have $\psi_{n, \lambda}(S) \psi_{m, \mu}(T)=\psi_{m, \mu}(T) \psi_{n, \lambda}(S)$. Taking a limit as $n \rightarrow+\infty$ and $m \rightarrow+\infty$, we derive (P4).

\section{Examples and remarks}

Example 8.1. Example 2.8 can be generalized using examples from [Gorin and Karahanjan 1977] and techniques from [Evgrafov 1979].

There is an operator for which (2-1) does not hold, whose asymptotic behavior satisfies $e^{i t A} \sim \rho e^{|t|^{\rho}}$ as $|t| \rightarrow \infty$ for every $\rho>1 / 4$.

Let $E$ be a vector space of entire functions, such that there exists $C \in \mathbb{R}$ such that $|f(z)| \leqslant C e^{\sigma|z|^{\rho}}$. Like in Example 2.8, $E$ is a Banach space if the norm $\|f\|$ of a function $f$ is defined as the smallest constant $C$ for which the inequality above holds. Since

$$
f^{\prime}(z)=\frac{1}{2 \pi i} \int_{|\xi|=1} \frac{f(\xi+z)}{\xi^{2}} d \xi,
$$

it follows that the operator $A=-i d / d z$ is a bounded linear operator on $E$; see [Gorin and Karahanjan 1977]. Hence

$$
\left(e^{i t A} f\right)(z)=\left(e^{t d / d z} f\right)(z)=f(z+t) .
$$

It is easy to see that $\left\|e^{i t A}\right\|=O\left(e^{|t|^{\rho}}\right)$ as $|t| \rightarrow \infty$.

To see exact asymptotic behavior, we can use a function

$$
f_{0}(z)=\sum_{n=0}^{\infty} \frac{z^{n}}{\Gamma(n / \rho+1)}
$$

( $\Gamma$ is the gamma function). Using that $f_{0}(x) \sim \rho e^{x^{\rho}}$ when $x \rightarrow \infty$ for $\rho>1 / 4$ (see [Evgrafov 1979, Example 1.5]), we derive our statement.

Example 8.2. Consider the operator $A=-i d / d x$ acting on the dense subspace of $L^{p}(\mathbb{R})$ consisting of all absolutely continuous functions whose derivative also belongs to $L^{p}(\mathbb{R})$. It is easy to see that $e^{i t A} f=f(x+t)$, and we can conclude $\left\|e^{i t A}\right\|=1$. Hence, for the operator $A$, we have the $F_{0}$ functional calculus. 
Example 8.3. Let $H$ be a Hilbert space and let $A$ be an unbounded selfadjoint operator. Also, let $Q$ be a nilpotent operator of order $n$, that is, $Q^{n}=0$, and $Q^{n-1} \neq 0$. Let $Q$ commute with $A$. Such a $Q$ is given by the matrix

$$
Q=\left[\begin{array}{ccccc}
0 & 1 & 0 & \cdots & 0 \\
0 & 0 & 1 & \cdots & 0 \\
\vdots & \vdots & \ddots & \ddots & 0 \\
\vdots & \vdots & \ddots & \ddots & 1 \\
0 & 0 & \cdots & \cdots & 0
\end{array}\right]
$$

with respect to the root subspace of some eigenvalue of $A$. Then we have

$$
\left\|e^{i t(A+Q)}\right\|=\left\|e^{i t A} e^{i t Q}\right\|=\left\|e^{i t Q}\right\|=O\left(|t|^{n-1}\right) .
$$

Therefore, for the operator $A+Q$, we have the $F_{n-1}$ functional calculus.

Example 8.4. The advection operator

$$
f(x, t) \mapsto a \cdot \nabla f(x, t)
$$

(here $\nabla=\left(\partial / \partial x_{1}, \ldots, \partial / \partial x_{n}\right), x \in \mathbb{R}^{k}$ and $\left.t \in \mathbb{R}\right)$ is well known in the mechanics of fluids. It is a generalized scalar operator in which $f(x, t)$ solves the abstract Cauchy problem $\partial f(x, t) / \partial t=a \cdot \nabla f(x, t)$. This unbounded operator commutes with the Laplacian. In fact, it commute with all $\partial / \partial x_{j}$. So we can construct an ultraweak functional calculus for these operators and finally we can get any linear (even some nonlinear) combinations of the advection operator and the Laplacian.

Finally, we shall see that the functional calculus we established contains, in a sense, the widest algebra of functions. We deal with the one dimensional case.

Theorem 8.5. The operator $A$ has the $F_{\alpha}$ functional calculus if and only if

$$
\left\|e^{i t A}\right\| \leqslant M\left(1+|t|^{\alpha}\right) \quad \text { for some } M \in \mathbb{R} .
$$

Proof. If there is $M \in \mathbb{R}$ such that $\left\|e^{i t A}\right\| \leqslant M\left(1+|t|^{\alpha}\right)$, then by Section $1, A$ has the $F_{\alpha}$ functional calculus.

In the opposite direction, since $d^{\alpha} e^{i t x} / d x^{\alpha}=(i t)^{\alpha} e^{i t x}$, it follows that

$$
\left\|e^{i t x}\right\|_{\alpha}=\sup _{x \in \mathbb{R}}\left|e^{i t x}\right|+\sup _{x \in \mathbb{R}}\left|\frac{d^{\alpha}}{d x^{\alpha}} e^{i t x}\right| \leqslant 1+|t|^{\alpha},
$$

and, from the existence of the functional calculus, it follows that

$$
\left\|\Phi\left(e^{i t x}\right)\right\| \leqslant M\left\|e^{i t x}\right\| \leqslant M\left(1+|t|^{\alpha}\right) .
$$




\section{Acknowledgements}

We are grateful to Professor Miloš Arsenović for help and many useful suggestions during the work on this paper. We also thank the referee for useful suggestions during the review process.

\section{References}

[Andersson 2003] M. Andersson, "(Ultra)differentiable functional calculus and current extension of the resolvent mapping”, Ann. Inst. Fourier (Grenoble) 53:3 (2003), 903-926. MR 2004g:47010 Zbl 1052.47009

[Andersson and Berndtsson 2002] M. Andersson and B. Berndtsson, "Non-holomorphic functional calculus for commuting operators with real spectrum", Ann. Sc. Norm. Super. Pisa Cl. Sci. (5) 1:4 (2002), 925-955. MR 2004d:47035 Zbl 1099.47505

[Andersson and Sjöstrand 2004] M. Andersson and J. Sjöstrand, "Functional calculus for noncommuting operators with real spectra via an iterated Cauchy formula", J. Funct. Anal. 210:2 (2004), 341-375. MR 2005b:47010 Zbl 1070.47009

[Andersson et al. 2006] M. Andersson, H. Samuelsson, and S. Sandberg, "Operators with smooth functional calculi”, J. Anal. Math. 98 (2006), 221-247. MR 2007g:47008 Zbl 1152.47010

[Balabane et al. 1993] M. Balabane, H. Emamirad, and M. Jazar, "Spectral distributions and generalization of Stone's theorem”, Acta Appl. Math. 31 (1993), 275-295. MR 94f:47038 Zbl 0802.47013

[Brackx et al. 1982] F. Brackx, R. Delanghe, and F. Sommen, Clifford analysis, Research Notes in Mathematics 76, Pitman, Boston, MA, 1982. MR 85j:30103 Zbl 0529.30001

[deLaubenfels 1991] R. deLaubenfels, "Existence and uniqueness families for the abstract Cauchy problem”, J. London Math. Soc. (2) 44:2 (1991), 310-338. MR 92k:34075 Zbl 0766.47011

[deLaubenfels 1993a] R. deLaubenfels, "Automatic well-posedness with the abstract Cauchy problem on a Fréchet space", J. London Math. Soc. (2) 48:3 (1993), 526-536. MR 94i:34119 Zbl 0792. 47042

[deLaubenfels 1993b] R. deLaubenfels, "Unbounded holomorphic functional calculus and abstract Cauchy problems for operators with polynomially bounded resolvents", J. Funct. Anal. 114 (1993), 348-394. MR 94h:47029 Zbl 0785.47018

[deLaubenfels 1994] R. deLaubenfels, Existence families, functional calculi and evolution equations, Lecture Notes in Mathematics 1570, Springer, Berlin, 1994. MR 96b:47047 Zbl 0811.47034

[deLaubenfels 1995] R. deLaubenfels, "Automatic extensions of functional calculi", Studia Math. 114:3 (1995), 237-259. MR 96f:47029 Zbl 0834.47012

[deLaubenfels and Jazar 1999] R. deLaubenfels and M. Jazar, "Functional calculi, regularized semigroups and integrated semigroups", Studia Math. 132:2 (1999), 151-172. MR 99k:47036

[Dunford and Schwartz 1958] N. Dunford and J. T. Schwartz, Linear Operators, I: General Theory, Wiley, New York, 1958. MR 1009162 (90g:47001a) Zbl 0084.10402

[Dynkin 1972] E. M. Dyn'kin, “An operator calculus based on the Cauchy-Green formula, III: Investigations on linear operators and the theory of functions", Zap. Naučn. Sem. Leningrad. Otdel. Mat. Inst. Steklov. (LOMI) 30 (1972), 33-39. In Russian. MR 48 \#6982

[Evgrafov 1979] М. A. Evgrafov, Асимптотические оценки и целые функции, 3rd ed., Nauka, Moscow, 1979. MR 81b:30048 Zbl 0447.30016 
[Gorin and Karahanjan 1977] E. A. Gorin and M. I. Karahanjan, “Асимптотический вариант теореми Фуглида-Путнама о комутаторах для элементов Банаховых алгебр”, Mat. Zametki 22:2 (1977), 179-188. MR 57 \#1123

[Helffer and Sjöstrand 1989] B. Helffer and J. Sjöstrand, "Équation de Schrödinger avec champ magnétique et équation de Harper", pp. 118-197 in Schrödinger operators (Sønderborg, 1988), edited by H. Holden and A. Jensen, Lecture Notes in Phys. 345, Springer, Berlin, 1989. MR 91g: 35078 Zbl 0699.35189

[McIntosh and Pryde 1987] A. McIntosh and A. Pryde, "A functional calculus for several commuting operators", Indiana Univ. Math. J. 36:2 (1987), 421-439. MR 88i:47007 Zbl 0694.47015

[McIntosh et al. 1988] A. McIntosh, A. Pryde, and W. Ricker, "Comparison of joint spectra for certain classes of commuting operators", Studia Math. 88:1 (1988), 23-36. MR 89e:47006 Zbl 0665.47002

[Rudin 1973] W. Rudin, Functional analysis, McGraw-Hill, New York, 1973. MR 51 \#1315 Zbl 0253.46001

[Ryan 2003] J. Ryan, "Introductory Clifford analysis", preprint, 2003. arXiv math.CV/0303339

[Vasilescu 1982] F.-H. Vasilescu, Analytic functional calculus and spectral decompositions, Mathematics and its Applications (East European Series) 1, D. Reidel, Dordrecht, 1982. MR 85b:47016 Zbl 0495.47013

Received October 19, 2009. Revised June 21, 2010.

DRAGOLJUB KeČKIĆ

MATEMATIČKI FAKULTET

UNIVERZITET U BEOGRADU

STUDENTSKI TRG 16

11000 BEOGRAD

SERBIA

keckic@matf.bg.ac.rs

http://poincare.matf.bg.ac.rs/ keckic/

ĐORĐE KRTINIĆ

MATEMATIČKI FAKULTET

UNIVERZITET U BEOGRADU

STUDENTSKI TRG 16

11000 BEOGRAD

SERBIA

georg@matf.bg.ac.rs

http://poincare.matf.bg.ac.rs/ georg/ 


\title{
PACIFIC JOURNAL OF MATHEMATICS
}

\author{
http://www.pjmath.org \\ Founded in 1951 by \\ E. F. Beckenbach (1906-1982) and F. Wolf (1904-1989)
}

\section{EDITORS}

V. S. Varadarajan (Managing Editor)

Department of Mathematics

University of California

Los Angeles, CA 90095-1555

pacific@math.ucla.edu

Vyjayanthi Chari

Department of Mathematics

University of California

Riverside, CA 92521-0135

chari@math.ucr.edu

Robert Finn

Department of Mathematics Stanford University

Stanford, CA 94305-2125

finn@math.stanford.edu

Kefeng Liu

Department of Mathematics

University of California

Los Angeles, CA 90095-1555

liu@math.ucla.edu
Darren Long

Department of Mathematics

University of California

Santa Barbara, CA 93106-3080

long@math.ucsb.edu

Jiang-Hua Lu

Department of Mathematics

The University of Hong Kong

Pokfulam Rd., Hong Kong jhlu@maths.hku.hk

Alexander Merkurjev

Department of Mathematics

University of California

Los Angeles, CA 90095-1555

merkurev@math.ucla.edu
Sorin Popa

Department of Mathematics University of California

Los Angeles, CA 90095-1555 popa@math.ucla.edu

Jie Qing

Department of Mathematics

University of California

Santa Cruz, CA 95064

qing@cats.ucsc.edu

Jonathan Rogawski

Department of Mathematics

University of California

Los Angeles, CA 90095-1555

jonr@math.ucla.edu

\section{PRODUCTION}

pacific@math.berkeley.edu

\begin{abstract}
Silvio Levy, Scientific Editor Matthew Cargo, Senior Production Editor
\end{abstract}
ACADEMIA SINICA, TAIPEI

CALIFORNIA INST. OF TECHNOLOGY

INST. DE MATEMÁTICA PURA E APLICADA

KEIO UNIVERSITY

MATH. SCIENCES RESEARCH INSTITUTE

NEW MEXICO STATE UNIV.

OREGON STATE UNIV.

\section{SUPPORTING INSTITUTIONS}

STANFORD UNIVERSITY
UNIV. OF BRITISH COLUMBIA
UNIV. OF CALIFORNIA, BERKELEY
UNIV. OF CALIFORNIA, DAVIS
UNIV. OF CALIFORNIA, LOS ANGELES
UNIV. OF CALIFORNIA, RIVERSIDE
UNIV. OF CALIFORNIA, SAN DIEGO
UNIV. OF CALIF., SANTA BARBARA

UNIV. OF CALIF., SANTA CRUZ

UNIV. OF MONTANA

UNIV. OF OREGON

UNIV. OF SOUTHERN CALIFORNIA

UNIV. OF UTAH

UNIV. OF WASHINGTON

WASHINGTON STATE UNIVERSITY

These supporting institutions contribute to the cost of publication of this Journal, but they are not owners or publishers and have no responsibility for its contents or policies.

See inside back cover or www.pjmath.org for submission instructions.

The subscription price for 2011 is US \$420/year for the electronic version, and \$485/year for print and electronic.

Subscriptions, requests for back issues from the last three years and changes of subscribers address should be sent to Pacific Journal of Mathematics, P.O. Box 4163, Berkeley, CA 94704-0163, U.S.A. Prior back issues are obtainable from Periodicals Service Company, 11 Main Street, Germantown, NY 12526-5635. The Pacific Journal of Mathematics is indexed by Mathematical Reviews, Zentralblatt MATH, PASCAL CNRS Index, Referativnyi Zhurnal, Current Mathematical Publications and the Science Citation Index.

The Pacific Journal of Mathematics (ISSN 0030-8730) at the University of California, c/o Department of Mathematics, 969 Evans Hall, Berkeley, CA 94720-3840, is published monthly except July and August. Periodical rate postage paid at Berkeley, CA 94704, and additional mailing offices. POSTMASTER: send address changes to Pacific Journal of Mathematics, P.O. Box 4163, Berkeley, CA 94704-0163.

PJM peer review and production are managed by EditFLOW ${ }^{\mathrm{TM}}$ from Mathematical Sciences Publishers.

PUBLISHED BY PACIFIC JOURNAL OF MATHEMATICS

at the University of California, Berkeley 94720-3840

A NON-PROFIT CORPORATION

Typeset in LATEX

Copyright $(02011$ by Pacific Journal of Mathematics 


\section{PACIFIC JOURNAL OF MATHEMATICS}

Volume $249 \quad$ No. $1 \quad$ January 2011

Metabelian SL $(n, \mathbb{C})$ representations of knot groups, II: Fixed points

HANS U. BODEN and STEFAN FRIEDL

Lewis-Zagier correspondence for higher-order forms

ANTON DEITMAR

Topology of positively curved 8-dimensional manifolds with symmetry

ANAND DESSAI

Strong Kähler with torsion structures from almost contact manifolds

MARISA FERnÁNDEZ, ANNA FINO, LUIS UGARTE and RAQUEL

VILLACAMPA

Connections between Floer-type invariants and Morse-type invariants of Legendrian knots

MICHAEL B. HENRY

A functional calculus for unbounded generalized scalar operators on Banach spaces

DRAGOLJUB KEČKIĆ and ĐORĐE KRTINIĆ

Geometric formality of homogeneous spaces and of biquotients

D. KoTSCHICK and S. TERZIĆ

Positive solutions for a nonlinear third order multipoint boundary value problem

Yang LiU, Zhang Weiguo, Liu Xiping, Shen Chunfang and Chen HUA

The braid group surjects onto $G_{2}$ tensor space

SCOTT MORRISON

Analogues of the Wiener Tauberian and Schwartz theorems for radial functions on symmetric spaces

E. K. NARAyAnAn and Alladi Sitaram

Semidirect products of representations up to homotopy

Yunhe SHENG and Chenchang ZHU

Homology sequence and excision theorem for Euler class group

YONG YANG 